\title{
LEFT AND RIGHT GENERALIZED DRAZIN INVERTIBILITY OF AN UPPER TRIANGULAR OPERATOR MATRICES WITH APPLICATION TO BOUNDARY VALUE PROBLEMS
}

\section{KOUIDER MILOUD HOCINE ${ }^{1}$, BEKKAI MESSIRDI ${ }^{1}$ AND MOHAMMED BENHARRAT ${ }^{2, *}$}

\author{
${ }^{1}$ Laboratoire de Mathématiques Fondamentales et Appliquées d'Oran (LMFAO), Département de \\ Mathématiques, Université d'Oran 1 Ahmed Ben Bella; 31000 Oran, Algérie \\ ${ }^{2}$ Laboratoire de Mathématiques Fondamentales et Appliquées d'Oran (LMFAO), Département de \\ Mathématiques et informatique, Ecole National Polytechnique d'Oran-Maurice Audin (Ex. ENSET \\ d'Oran); B.P. 1523 Oran-El M'Naouar-, Oran, Algérie
}

*Corresponding author: mohammed.benharrat@gmail.com

\begin{abstract}
When $A \in \mathcal{B}(H)$ and $B \in \mathcal{B}(K)$ are given, we denote by $M_{C}$ the operator on the Hilbert space $H \oplus K$ of the form $M_{C}=\left(\begin{array}{cc}A & C \\ 0 & B\end{array}\right)$. In this paper we investigate the quasi-nilpotent part and the analytical core for the upper triangular operator matrix $M_{C}$ in terms of those of $A$ and $B$. We give some necessary and sufficient conditions for $M_{C}$ to be left or right generalized Drazin invertible operator for some $C \in \mathcal{B}(K, H)$. As an application, we study the existence and uniqueness of the solution for abstract boundary value problems described by upper triangular operator matrices with right generalized Drazin invertible component.
\end{abstract}

Received 2017-10-26; accepted 2018-01-04; published 2019-01-04.

2010 Mathematics Subject Classification. 47A10, 47B38.

Key words and phrases. generalized drazin inverse; left generalized drazin inverse; right generalized drazin inverse; upper triangular operator matrices.

(C)2019 Authors retain the copyrights of their papers, and all open access articles are distributed under the terms of the Creative Commons Attribution License. 


\section{INTRODUCTION AND PRELIMINARIES}

Let $\mathcal{B}(H)$ be the Banach algebra of all bounded linear operators acting on an infinite-dimensional complex Hilbert space $H$. Associated with an operator $T$ there are two (not necessarily closed) linear subspaces of $H$ invariant by $T$, played an important role in the development of the generalized Drazin inverse for $T \in \mathcal{B}(H)$, the quasi-nilpotent part $H_{0}(T)$ of $T$ :

$$
H_{0}(T)=\left\{x \in H: \lim _{n \rightarrow \infty}\left\|T^{n} x\right\|^{\frac{1}{n}}=0\right\}
$$

and the analytical core $K(T)$ of $T$ :

$$
K(T)=\left\{x \in H: \text { there exist a sequence }\left(x_{n}\right) \text { in } H \text { and a constant } \delta>0\right.
$$

such that $T x_{1}=x, T x_{n+1}=x_{n}$ and $\left\|x_{n}\right\| \leq \delta^{n}\|x\|$ for all $\left.n \in \mathbb{N}\right\}$.

See also [1]. It is well-known that if $K(T)$ and $H_{0}(T)$ are both closed, $H=H_{0}(T) \oplus K(T)$, the restriction of $T$ to $H_{0}(T)$ is a quasi-nilpotent operator, and the restriction of $T$ to $K(T)$ is invertible, provided that $T$ is generalized Drazin invertible, (c.f. [18]). Recently, by the use of this two subspaces, in [24], the authors defined and studied a new class of operators called left and right generalized Drazin invertible operators as a generalization of left and right Drazin invertible operators.

Definition 1.1. An operator $T \in \mathcal{B}(H)$ is said to be right generalized Drazin invertible if $K(T)$ is closed and complemented with a subspace $N$ in $H$ such that $T(N) \subset N \subseteq H_{0}(T)$.

Definition 1.2. An operator $T \in \mathcal{B}(H)$ is said to be left generalized Drazin invertible if $H_{0}(T)$ is closed and complemented with a subspace $M$ in $H$ such that $T(M) \subset M$ and $T(M)$ is closed.

We also proved that $T \in \mathcal{B}(H)$ is a right generalized Drazin invertible if and only if 0 is an isolated point on the surjective spectrum $\sigma_{s u}(T)$ of $T$ and by duality $T \in \mathcal{B}(H)$ is a left generalized Drazin invertible if and only if 0 is an isolated point on the approximate spectrum $\sigma_{a p}(T)$. So we are mainly interested in the case where the point 0 belongs to the spectrum $\sigma(T)$ of $T$ or in its various distinguished parts. Here, we are interested in the analogous question for an upper triangular operator of the form

$$
M_{C}=\left(\begin{array}{cc}
A & C \\
0 & B
\end{array}\right) \text {. }
$$

defined on the separable Hilbert space $H \oplus K$. Recall that the problem of the relationship between the spectrum, various distinguished parts of the spectrum of an upper triangular and Drazin invertibility and its diagonal has been considered by a number of authors in the recent past, we can see $[4,5,13,21,22,28-30]$ and the references therein for recent reviews on this topic. A related, and seemingly more demanding, problem 
is the following. Let $H$ be a Hilbert space, $T$ is a bounded linear operator on $H$, and $M$ is a $T$-invariant closed subspace of $H$, then $T$ takes the form

$$
T=\left(\begin{array}{ll}
* & * \\
0 & *
\end{array}\right): M \oplus M^{\perp} \rightarrow M \oplus M^{\perp}
$$

which motivated the interest in $2 \times 2$ upper-triangular operator matrices.

In this paper we use the results of [24] to give a necessary and sufficient conditions for $M_{C}$ to be left (resp. right) generalized Drazin invertible which generalizes the notion of generalized Drazin invertible operators to the matrix case. We characterize the quasi-nilpotent part and the analytical core of the operator $M_{C}$ in term of the pair $(A, B)$ of bounded operators. We apply our results to study the existence and uniqueness of solutions of boundary value problems described by an upper triangular operator matrices $(2 \times 2)$ acting in Hilbert spaces with a complex spectral parameter $\lambda$ :

$$
(\mathcal{P})\left\{\begin{array}{l}
\left(U_{L}-\lambda M_{C}\right) w=F \\
\Gamma w=\Phi
\end{array},\right.
$$

where $U_{L}$ is right generalized Drazin invertible, $\Gamma$ is a boundary operator, $F$ and $\Phi$ are given.

For $T \in \mathcal{B}(H)$ write $N(T), R(T), \sigma(T)$ and $\rho(T)$ respectively, the null space, the range, the spectrum and the resolvent set of $T$. The nullity and the deficiency of $T$ are defined respectively by $\alpha(T)=\operatorname{dim} N(T)$ and $\beta(T)=\operatorname{dim} H / R(T)$. Here $I$ denotes the identity operator in $H$. By iso $\sigma(T)$ and acc $\sigma(T)$ we define the set of all isolated and accumulation spectral points of $T$.

If $M$ is a subspace of $H$ then $T_{M}$ denote the restriction of $T$ in $M$. Assume that $M$ and $N$ are two subspaces of $H$ such that $H=M \oplus N$ (that is $H=M+N$ and $M \cap N=0$ ). We say that $T$ is completely reduced by the pair $(M, N)$, denoted as $(M, N) \in \operatorname{Red}(T)$, if $T(M) \subset M, T(N) \subset N$ and $T=T_{M} \oplus T_{N}$. In such case we have $N(T)=N\left(T_{M}\right) \oplus N\left(T_{N}\right), R(T)=R\left(T_{M}\right) \oplus R\left(T_{N}\right)$ and $T^{n}=T_{M}^{n} \oplus T_{N}^{n}$ for all $n \in \mathbb{N}$. An operator is said to be bounded below if it is injective with closed range.

Recall that (see, e.g. [14]) the ascent $a(T)$ of an operator $T \in \mathcal{B}(H)$ is defined as the smallest nonnegative integer $p$ such that $N\left(T^{p}\right)=N\left(T^{p+1}\right)$. If no such an integer exists, we set $a(T)=\infty$. Analogously, the smallest nonnegative integer $q$ such that $R\left(T^{q}\right)=R\left(T^{q+1}\right)$ is called the descent of $T$ and denoted by $d(T)$. We set $d(T)=\infty$ if for each $q, R\left(T^{q+1}\right)$ is a proper subspace of $R\left(T^{q}\right)$. It is well known that if the ascent and the descent of an operator are finite, then they are equal. Furthermore, if $a(T)=p<\infty$ then $H_{0}(T)=N\left(T^{p}\right)$ and if $d(T)=q<\infty$ then $K(T)=R\left(T^{q}\right)$.

An operator $T \in \mathcal{B}(H)$ is said to be Drazin invertible, if there exists an operator $S \in \mathcal{B}(H)$ such that

$$
S T=T S \quad S T S=S \text { and } T S T=T+U \text { where } U \text { is a nilpotent operator. }
$$


The concept of Drazin invertible operators has been generalized by Koliha ( [18]) by replacing the nilpotent operator $U$ in (1.2) by a quasi-nilpotent operator one. In this case, $S$ is called a generalized Drazin inverse of $T$, denoted by $T^{D}$. Examples of generalized Drazin invertible operators are the operators of the following classes:

- Invertible operators, right invertible operators and left invertible operators.

- Left Drazin invertible operators,

$$
L D(H)=\left\{T \in \mathcal{B}(H): a(T) \text { is finite and } R\left(T^{a(T)+1}\right) \text { is closed }\right\} .
$$

- Right Drazin invertible operators,

$$
R D(H)=\left\{T \in \mathcal{B}(H): d(T) \text { is finite and } R\left(T^{d(T)}\right) \text { is closed }\right\}
$$

- Drazin invertible operators,

$$
L D(H) \cap R D(H) .
$$

According to the Definitions 1.1 and 1.2, we also have

Right (resp. Left) invertible operator $\Longrightarrow$ Right (resp. Left) Drazin invertible operator $\Longrightarrow$ Right (resp. Left) generelazed Drazin invertible operator.

The left Drazin spectrum, the right Drazin spectrum, the Drazin spectrum, the generalized Drazin spectrum, the left generalized Drazin spectrum and the right generalized Drazin spectrum of $T$ are, respectively, defined by

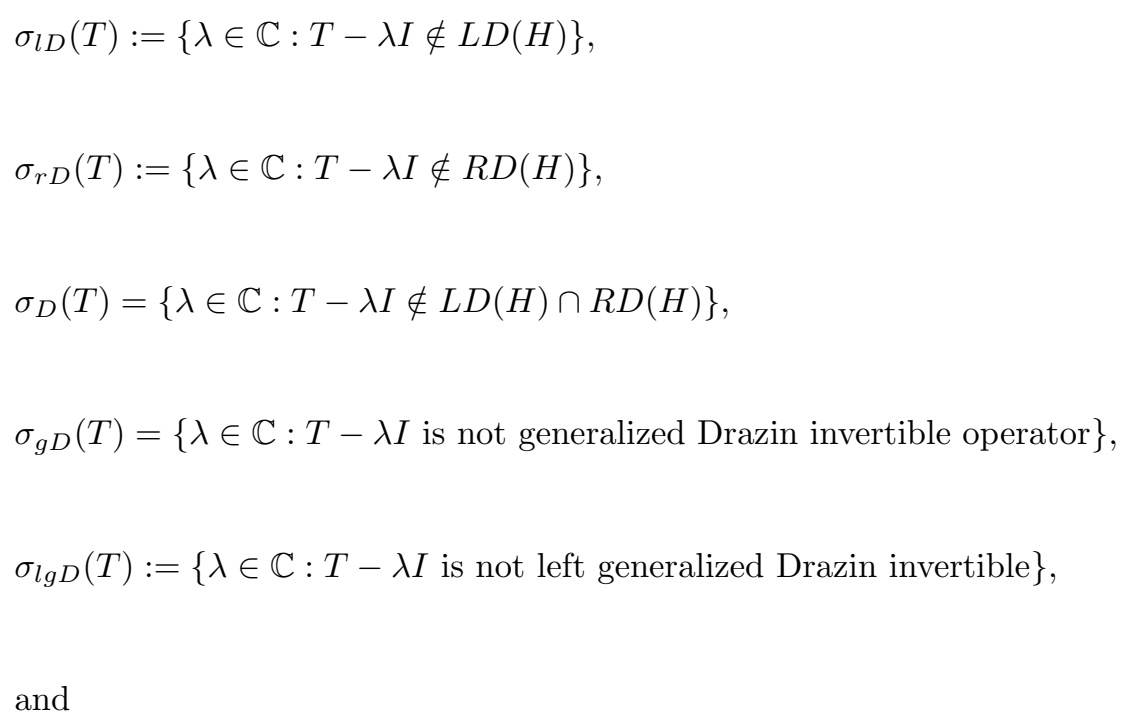


It is well known that these spectra are compact sets in the complex plane, and we have,

$$
\begin{gathered}
\sigma_{g D}(T)=\sigma_{l g D}(T) \cup \sigma_{r g D}(T) \subset \sigma_{D}(T)=\sigma_{l D}(T) \cup \sigma_{r D}(T), \\
\sigma_{l g D}(T) \subset \sigma_{l D}(T) \subset \sigma_{a p}(T),
\end{gathered}
$$

and

$$
\sigma_{r g D}(T) \subset \sigma_{r D}(T) \subset \sigma_{s u}(T)
$$

where

$$
\sigma_{a p}(T):=\{\lambda \in \mathbb{C}: T-\lambda I \text { is not bounded below }\}
$$

and

$$
\sigma_{s u}(T):=\{\lambda \in \mathbb{C}: T-\lambda I \text { is not surjective }\}
$$

are respectively the approximate point spectrum and the surjective spectrum of $T$.

The basic existence results of generalized Drazin inverses and its relation to the quasi-nilpotent part and the analytical core are summarized in the following theorems.

Theorem 1.1 ( [18]). Assume that $T \in \mathcal{B}(H)$. The following assertions are equivalent:

(i) $T$ is generalized Drazin invertible,

(ii) 0 is an isolated point in the spectrum $\sigma(T)$ of $T$;

(iii) $K(T)$ is closed and $H=K(T) \oplus H_{0}(T)$,

(iv) $H_{0}(T)$ is closed and $H=K(T) \oplus H_{0}(T)$,

(v) there is a bounded projection $P$ on $H$ such that $R(P)=K(T)$ and $N(P)=H_{0}(T)$.

(vi) $T=T_{1} \oplus T_{2}$, with $T_{1}=T_{K(T)}$ is invertible operator and $T_{2}=T_{H_{0}(T)}$ is quasi-nilpotent operator.

$\oplus$ denotes the algebraic direct sum and $T_{M}$ denote the restriction of $T$ to a subspace $M$ of $H$.

Theorem 1.2. Assume that $T \in \mathcal{B}(H)$. The following assertions are equivalent:

(i) $T$ is left generalized Drazin invertible;

(ii) 0 is an isolated point in $\sigma_{a p}(T)$;

(iii) $T=T_{1} \oplus T_{2}$, with $T_{1}=T_{M}$ is left invertible operator and $T_{2}=T_{H_{0}(T)}$ is quasi-nilpotent operator.

Proof. The equivalence $[(\mathrm{i})] \Longleftrightarrow[($ iii) $]$ follows by [24, Proposition 3.2] and the implication [(i)] $\Longrightarrow[(i i)]$ follows from [24, Theorem 3.8]. Now, if 0 is an isolated point in $\sigma_{a p}(T)$, then by [23, Theorem 4.4] 0 is a singularity of the generalized resolvent, equivalently, $T$ admits a generalized Kato decomposition $(M, N)$, and since $T$ has the SVEP at 0 , it follows from [1, Theorem 3.14] that $T_{M}$ is injective and $H_{0}(T)=N$. This proved the implication $[($ ii) $] \Longrightarrow[(i)]$ and hence $[(i)] \Longleftrightarrow[(i i)]$. 
We know that the properties to be right generalized Drazin invertible or to be left generalized Drazin invertible are dual each other, (see [24, Proposition 3.9]), then we have,

Theorem 1.3. Let $T \in \mathcal{B}(H)$. The following assertions are equivalent:

(i) $T$ is right generalized Drazin invertible;

(ii) 0 is an isolated point in $\sigma_{s u}(T)$;

(iii) $T=T_{1} \oplus T_{2}$, with $T_{1}=T_{K(T)}$ is right invertible operator and $T_{2}=T_{N}$ is quasi-nilpotent operator.

The reduced minimum modulus $\gamma(T)$ of $T$ is defined by

$$
\gamma(T)= \begin{cases}\inf \{\|T x\|: \operatorname{dis}(x, N(T))=1\} & \text { if } T \neq 0 \\ 0 & \text { if } T=0\end{cases}
$$

and $\operatorname{dis}(x, N(T))=\inf \{\|x-z\|$ such that $z \in N(T)\}$. It is well known that $\gamma(T)>0$ if and only if $R(T)$ is closed.

The paper is organized as follows. In Section 2 we give a relationship between the quasi-nilpotent part and the analytical core of a pair $(A, B)$ of operators and that of $2 \times 2$ block triangular matrices $M_{C}$, we show that the quasi-nilpotent part of $M_{C}$ is a direct sum of the quasi-nilpotent part of $A$ and $B$ and that is the same for analytical core. In Section 3, we study the left (resp. right) generalized Drazin invertibility of $M_{C}$ using the isolated point in the approximate spectrum (resp. the surjective spectrum) of $A$ and $B$. Finally in section 4 we illustrate our approach by studying a boundary value problems described by an upper triangular operator matrices.

\section{The quasi-nilpotent Part And the Analytical Core of The operator $M_{C}$}

In the following, we find the relationship between the quasi-nipotent part (resp. the analytical core) of the pair $(A, B)$ of operators and that of $M_{C}$ defined in (1.1) and we give fundamental results concerning this operator.

Proposition 2.1. If $R(C) \subset H_{0}(A)$, then

$$
H_{0}\left(M_{C}\right)=H_{0}(A) \oplus H_{0}(B) .
$$

Proof. Suppose that $\left(\begin{array}{l}x \\ y\end{array}\right) \in H_{0}(A) \oplus H_{0}(B)$ and consider $H \times K$ the product Hilbert space equipped with the norm

$$
\left\|\left(\begin{array}{l}
x \\
y
\end{array}\right)\right\|^{2}=\|x\|^{2}+\|y\|^{2} .
$$


We have

$$
\left\|M_{C}^{n}\left(\begin{array}{l}
x \\
y
\end{array}\right)\right\|^{2}=\left\|A^{n} x+S y\right\|^{2}+\left\|B^{n} y\right\|^{2},
$$

with

$$
S=A^{n-1} C+A^{n-2} C B+\cdots+A C B^{n-2}+C B^{n-1} .
$$

Then

$$
\left\|M_{C}^{n}\left(\begin{array}{l}
x \\
y
\end{array}\right)\right\|^{\frac{2}{n}} \leq\left\|A^{n} x\right\|^{\frac{2}{n}}+\left\|B^{n} y\right\|^{\frac{2}{n}}+\|S y\|^{\frac{2}{n}}
$$

Therefore,

$$
\|S\|^{\frac{2}{n}} \leq \sum_{p+k=n-1}\left\|A^{p} C B^{k} y\right\|^{\frac{2}{n}} .
$$

If $p \rightarrow \infty$ then $n \rightarrow \infty$ and since $R(C) \subset H_{0}(A)$, we obtain $\lim _{n \rightarrow \infty}\|S\|^{\frac{2}{n}}=0$. It follows that

$$
\begin{aligned}
& \lim _{n \rightarrow \infty}\left\|M_{C}^{n}\left(\begin{array}{l}
x \\
y
\end{array}\right)\right\|^{\frac{2}{n}}=0 \text {. Hence }\left(\begin{array}{l}
x \\
y
\end{array}\right) \in H_{0}\left(M_{C}\right) . \\
& \quad \text { Let now }\left(\begin{array}{l}
x \\
y
\end{array}\right) \in H_{0}\left(M_{C}\right) \text {. It is clear that }\left\|M_{C}^{n}\left(\begin{array}{l}
x \\
0
\end{array}\right)\right\|^{\frac{2}{n}}=\left\|A^{n} x\right\|^{\frac{2}{n}} \text { and }\left\|M_{C}^{n}\left(\begin{array}{l}
0 \\
y
\end{array}\right)\right\|^{\frac{2}{n}} \geq\left\|B^{n} y\right\|^{\frac{2}{n}} .
\end{aligned}
$$

Then $\lim _{n \rightarrow \infty}\left\|A^{n} x\right\|^{\frac{2}{n}}=0$ and $\lim _{n \rightarrow \infty}\left\|B^{n} y\right\|^{\frac{2}{n}}=0$. Thus $x \in H_{0}(A)$ and $y \in H_{0}(B)$.

Proposition 2.2. If $R(B) \subset N(C)$, then

$$
K\left(M_{C}\right)=K(A) \oplus K(B) .
$$

Proof. Let $x \in K(A)$ and $y \in K(B)$, by definition there exist two sequences $\left(x_{n}\right)$ in $H,\left(y_{n}\right)$ in $K$ and a constants $\delta_{1}>0, \delta_{2}>0$ such that $A x_{1}=x, A x_{n+1}=x_{n}$ and $\left\|x_{n}\right\| \leq \delta_{1}^{n}\|x\|$ and $B y_{1}=y, B y_{n+1}=y_{n}$ and $\left\|y_{n}\right\| \leq \delta_{2}^{n}\|y\|$ for all $n \in \mathbb{N}$.

We have

$$
M_{C}\left(\begin{array}{c}
x_{n+1} \\
y_{n+1}
\end{array}\right)=\left(\begin{array}{c}
A x_{n+1}+C y_{n+1} \\
B y_{n+1}
\end{array}\right)=\left(\begin{array}{c}
x_{n}+C B y_{n+2} \\
y_{n}
\end{array}\right) .
$$

Since $R(B) \subset N(C)$, it follows that

$$
M_{C}\left(\begin{array}{c}
x_{n+1} \\
y_{n+1}
\end{array}\right)=\left(\begin{array}{c}
x_{n} \\
y_{n}
\end{array}\right) \text { and } M_{C}\left(\begin{array}{l}
x_{1} \\
y_{1}
\end{array}\right)=\left(\begin{array}{l}
x \\
y
\end{array}\right) .
$$

Furthermore

$$
\left\|\left(\begin{array}{l}
x_{n} \\
y_{n}
\end{array}\right)\right\| \leq \delta^{n}\left\|\left(\begin{array}{l}
x \\
y
\end{array}\right)\right\|
$$

where $\delta=\max \left(\delta_{1}, \delta_{2}\right)$. Hence $\left(\begin{array}{l}x \\ y\end{array}\right) \in K\left(M_{C}\right)$. 
Conversely, suppose that $\left(\begin{array}{l}x \\ y\end{array}\right) \in K\left(M_{C}\right)$. Then there exist a sequence $\left(\begin{array}{c}x_{n} \\ y_{n}\end{array}\right)$ in $H \times K$ and a constant $\delta>0$ such that $M_{C}\left(\begin{array}{c}x_{1} \\ y_{1}\end{array}\right)=\left(\begin{array}{c}x \\ y\end{array}\right), M_{C}\left(\begin{array}{c}x_{n+1} \\ y_{n+1}\end{array}\right)=\left(\begin{array}{c}x_{n} \\ y_{n}\end{array}\right)$ and $\left\|\left(\begin{array}{c}x_{n} \\ y_{n}\end{array}\right)\right\| \leq \delta^{n}\left\|\left(\begin{array}{c}x \\ y\end{array}\right)\right\|$ for all $n \in \mathbb{N}$.

We obtain from (2.1) that $B y_{n+1}=y_{n}, A x_{n+1}=x_{n}, A x_{1}=x$ and $B y_{1}=y$.

Consequently, $\left\|x_{n}\right\| \leq \delta^{n}\|x\|$ and $\left\|y_{n}\right\| \leq \delta^{n}\|y\|$, that is $\left(\begin{array}{l}x \\ y\end{array}\right) \in K(A) \oplus K(B)$. This completes the proof.

As a consequence of Propositions 2.1 and 2.2 we have the following result.

Corollary 2.1. If $R(C) \subset H_{0}(A)$ and $R(B) \subset N(C)$. Then $M_{C}$ is generalized Drazin invertible if and only if both $A$ and $B$ are generalized Drazin invertible.

\section{Left And Right Generalized Drazin invertibility of $M_{C}$}

Hwang and Lee, [13], give a necessary and sufficient condition for $M_{C}$ to be bounded below for some $C \in \mathcal{B}(K, H)$ and they are characterized the intersection of the approximate point spectrum, the surjective spectrum and the spectrum of $M_{C}$.

The next theorem is an extension of [13, Theorem 1], we will give some necessary and sufficient conditions for $M_{C}$ to be left generalized Drazin invertible operator for some $C \in \mathcal{B}(K, H)$.

Theorem 3.1. For a given pair $(A, B)$ of bounded operators, the following statements are equivalent:

(i) $M_{C}$ is left generalized Drazin invertible for some $C \in \mathcal{B}(K, H)$,

(ii) $A$ is left generalized Drazin invertible and there exists a constant $\delta$ such that

$$
\begin{cases}\alpha(B-\lambda I) \leq \beta(A-\lambda I) & \text { if } R(B-\lambda I) \text { is closed, } \\ \text { or } & \\ \beta(A-\lambda I)=\infty & \text { if } R(B-\lambda I) \text { is not closed, }\end{cases}
$$

To prove this theorem we need the following lemma.

Lemma 3.1. Let $T_{1}, T_{2}$ and $T_{3} \in \mathcal{B}(H)$ be ginven such that $T_{2}$ is invertible. If $\alpha\left(T_{1}\right)<\infty$ and $R\left(T_{1} T_{2} T_{3}\right)$ is closed, then $R\left(T_{3}\right)$ is also closed.

Proof. Follows from [12, Theorem 1].

Proof of Theorem 3.1. We first claim that if $A$ is left generalized Drazin invertible and there exists a constant $\delta$ such that for every $\lambda$ with $0<|\lambda|<\delta, R(B-\lambda I)$ is closed, then $\alpha(B-\lambda I) \leq \beta(A-\lambda I)$ is equivalent 
to $M_{C}$ is left generalized Drazin invertible for some $C \in \mathcal{B}(K, H)$. Indeed, since $A$ is left generalized Drazin invertible, then by Theorem 1.2, $A-\lambda I$ is bounded below for $0<|\lambda|<\delta$.

Assume that $\alpha(B-\lambda I) \leq \beta(A-\lambda I)$. Then there exists an isometry $J: N(B-\lambda I) \rightarrow R(A-\lambda I)^{\perp}$. Define an operator $C: K \rightarrow H$ by

$$
\begin{aligned}
& C:=\left(\begin{array}{ll}
J & 0 \\
0 & 0
\end{array}\right):\left(\begin{array}{c}
N(B-\lambda I) \\
N(B-\lambda I)^{\perp}
\end{array}\right) \rightarrow\left(\begin{array}{c}
R(A-\lambda I)^{\perp} \\
R(A-\lambda I)
\end{array}\right) . \\
& \text { Let }\left(\begin{array}{c}
x \\
y
\end{array}\right) \in N\left(M_{C}-\lambda I\right) \text {. Then } \\
& \left(M_{C}-\lambda I\right)\left(\begin{array}{c}
x \\
y
\end{array}\right)=\left(\begin{array}{c}
0 \\
0
\end{array}\right) ;
\end{aligned}
$$

implies that $(A-\lambda I) x+C y=0$ and $(B-\lambda I) y=0$. Since $A-\lambda I$ is injective and the fact that $N(C-\lambda I) \subseteq$ $N(B-\lambda I)^{\perp}$, we get $x=0$ and $y=0$. Then $M_{C}-\lambda I$ is injective.

Now we prove that $R\left(M_{C}-\lambda I\right)$ is closed. Let $\left(\begin{array}{l}x \\ y\end{array}\right) \in N\left(M_{C}-\lambda I\right)^{\perp}$. Then

$$
\begin{aligned}
\left\|\left(M_{C}-\lambda I\right)\left(\begin{array}{l}
x \\
y
\end{array}\right)\right\|^{2} & =\|(A-\lambda I) x+C y\|^{2}+\|(B-\lambda I) y\|^{2} \\
& =\|(A-\lambda I) x\|^{2}+\|C y\|^{2}+\|(B-\lambda I) y\|^{2} .
\end{aligned}
$$

Write $y:=y_{1}+y_{2}$, where $y_{1} \in N(B-\lambda I)$ and $y_{2} \in N(B-\lambda I)^{\perp}$. Then $\|C y\|=\left\|y_{1}\right\|,\|(A-\lambda I) x\| \geq$ $\gamma(A-\lambda I)\|x\|$ and $\left\|(B-\lambda I) y_{2}\right\| \geq \gamma(B-\lambda I)\left\|y_{2}\right\|$, because $R(B-\lambda I)$ and $R(A-\lambda I)$ are closed. Hence

$$
\begin{aligned}
\left\|\left(M_{C}-\lambda I\right)\left(\begin{array}{l}
x \\
y
\end{array}\right)\right\|^{2} & \geq \gamma^{2}(A-\lambda I)\|x\|^{2}+\left\|y_{1}\right\|^{2}+\gamma^{2}(B-\lambda I)\left\|y_{2}\right\|^{2} \\
& \geq \min \left(\gamma^{2}(A-\lambda I), \gamma^{2}(B-\lambda I), 1\right)\left\|\left(\begin{array}{l}
x \\
y
\end{array}\right)\right\|^{2} .
\end{aligned}
$$

Then $\gamma\left(M_{C}-\lambda I\right)>0$ and $\left(M_{C}-\lambda I\right)$ is bounded below for $0<|\lambda|<\delta$. It follows from Theorem 1.2 that $M_{C}$ is left generalized Drazin invertible.

Conversely, It suffices to show that if $(A-\lambda I)$ is bounded below with $\alpha(B-\lambda I)>\beta(A-\lambda I)$ for $\lambda \in \mathbb{C}$, then $\left(M_{C}-\lambda I\right)$ is not bounded below.

Assume that $\alpha(B-\lambda I)>\beta(A-\lambda I)$, so $\beta(A-\lambda I)<\infty$. We now consider the following two cases.

Case (1). If $N(C) \cap N(B-\lambda I) \neq\{0\}$. Then for all non-zero vector $z \in N(C) \cap N(B-\lambda I)$ we have $\left(M_{C}-\lambda I\right) z=0$. We conclude that $\left(M_{C}-\lambda I\right)$ is not bounded below.

Case (2). Suppose that $N(C) \cap N(B-\lambda I)=\{0\}$. Then $\operatorname{dim}(C(N(B-\lambda I)))=\alpha(B-\lambda I)>\beta(A-\lambda I)$. 
Thus, $C(N(B-\lambda I)) \cap R(A-\lambda I) \neq\{0\}$. We take a non-zero vector $z \in C(N(B-\lambda I)) \cap R(A-\lambda I)$. Then there exist some $x \in H$ and $y \in K$ such that $(A-\lambda I) x=C y=z$ and $(B-\lambda I) y=0$. Direct calculation shows that $\left(M_{C}-\lambda I\right)\left(\begin{array}{c}-x \\ y\end{array}\right)=0$. It follows that $\left(M_{C}-\lambda I\right)$ is not bounded below.

We next claim that if $A$ is left generalized Drazin invertible and there exists a constant $\delta$ such that for every $\lambda$ with $0<|\lambda|<\delta, R(B-\lambda I)$ is not closed, then $\beta(A-\lambda I)=\infty$ is equivalent to $M_{C}$ is left generalized Drazin invertible for some $C \in \mathcal{B}(K, H)$. Since $R(B-\lambda I)$ is not closed and $\beta(A-\lambda I)=\infty$, there exists an isomorphism $J: K \rightarrow R(B-\lambda I)$. Define an operator $C: K \rightarrow H$ in the following way:

$$
C:=\left(\begin{array}{ll}
J & 0
\end{array}\right): K \rightarrow\left(\begin{array}{c}
R(A-\lambda I)^{\perp} \\
R(A-\lambda I)
\end{array}\right)
$$

By a similar proof we check easily that $N\left(M_{C}-\lambda I\right)=\{0\}$ and $\gamma\left(M_{C}-\lambda I\right)>0$. That is $\left(M_{C}-\lambda I\right)$ is bounded below for $0<|\lambda|<\delta$ and by Theorem $1.2 M_{C}$ is left generalized Drazin invertible.

For the converse, suppose in the contrary that $\beta(A-\lambda I)<\infty$. Then

$$
\operatorname{dim} N\left(\left(\begin{array}{cc}
(A-\lambda I)^{*} & 0 \\
0 & I
\end{array}\right)\right)=\operatorname{dim} N\left((A-\lambda I)^{*}\right)=\beta(A-\lambda I)<\infty
$$

Since $R\left(\left(M_{C}-\lambda I\right)^{*}\right)$ is closed and $\left(\begin{array}{cc}I & 0 \\ C^{*} & I\end{array}\right)$ is invertible, by Lemma 3.1, we have that $R\left(\left(\begin{array}{cc}I & 0 \\ 0 & (B-\lambda I)^{*}\end{array}\right)\right)$ is closed, that is $R\left((B-\lambda I)^{*}\right)$ is closed. This contradicts our assumption. Therefore we must have $\beta(A-\lambda I)=\infty$.

By duality, we have:

Theorem 3.2. For a given pair $(A, B)$ of operators, the following statements are equivalent:

(i) $M_{C}$ is right generalized Drazin invertible for some $C \in \mathcal{B}(K, H)$,

(ii) $B$ is right generalized Drazin invertible and there exists a constant $\delta$ such that

$$
\begin{aligned}
& \begin{cases}\beta(A-\lambda I) \leq \alpha(B-\lambda I) & \text { if } R(A-\lambda I) \text { is closed, } \\
\text { or } & \\
\alpha(B-\lambda I)=\infty & \text { if } R(A-\lambda I) \text { is not closed, }\end{cases} \\
& \text { with } 0<|\lambda|<\delta .
\end{aligned}
$$

As a direct application of Theorem 3.1, the following corollary can be derived to give a characterization of $\sigma_{l g D}\left(M_{C}\right)$ for all $C \in \mathcal{B}(K, H)$. 


\section{Corollary 3.1.}

$$
\begin{aligned}
\bigcap_{C \in \mathcal{B}(K, H)} \sigma_{l g D}\left(M_{C}\right)= & \\
\sigma_{l g D}(A) & \bigcup\{\lambda \in \mathbb{C}: R(B-\lambda I) \text { is closed and } \beta(A-\lambda I)<\alpha(B-\lambda I)\} \\
& \bigcup\{\lambda \in \mathbb{C}: R(B-\lambda I) \text { is not closed and } \beta(A-\lambda I)<\infty\} .
\end{aligned}
$$

The following is the dual statement of Corollary 3.1.

\section{Corollary 3.2.}

$$
\begin{aligned}
\bigcap_{C \in \mathcal{B}(K, H)} \sigma_{r g D}\left(M_{C}\right)= & \\
\sigma_{r g D}(B) & \bigcup\{\lambda \in \mathbb{C}: R(A-\lambda I) \text { is closed and } \beta(A-\lambda I)>\alpha(B-\lambda I)\} \\
& \bigcup\{\lambda \in \mathbb{C}: \mathcal{R}(A-\lambda I) \text { is not closed and } \alpha(B-\lambda I)<\infty\} .
\end{aligned}
$$

By combining Corollaries 3.1 and 3.2 we obtain;

\section{Corollary 3.3.}

$$
\bigcap_{C \in \mathcal{B}(K, H)} \sigma_{g D}\left(M_{C}\right)=\sigma_{l g D}(A) \bigcup \sigma_{r g D}(B) \bigcup\{\lambda \in \mathbb{C}: \beta(A-\lambda I) \neq \alpha(B-\lambda I)\}
$$

This result gives a generalization of $[4$, Theorem 2.1].

\section{Application to a spectral boundary value matrix problem}

This section is devoted to the study of boundary value problems described by an upper triangular operator matrices $(2 \times 2)$ acting in Hilbert spaces with a complex spectral parameter $\lambda$,

$$
(\mathcal{P})\left\{\begin{array}{l}
\left(U_{L}-\lambda M_{C}\right) w=F \\
\Gamma w=\Phi
\end{array},\right.
$$

where $F$ and $\Phi$ are given and $U_{L}$ is the matrix operator defined on $H \oplus K$ by

$$
U_{L}=\left(\begin{array}{cc}
T & L \\
0 & D
\end{array}\right)
$$

with $L: K \rightarrow H$ a given linear operator. We first define the boundary value problem $(\mathcal{P})$ by ordered pairs $\left(U_{L}, M_{C}\right)$ of an upper triangular operator matrix $M_{C}$ where $U_{L}$ is a right generalized Drazin invertible and we construct the adapted boundary operator $\Gamma$ of $U_{L}$. We prove the existence of an unique solution of $(\mathcal{P})$ and we give an explicit expression for this solution. Before this down, we define the boundary operator for a right generalized Drazin invertible operator. 
If $S$ be a right generalized Drazin inverse of the operator $A \in \mathcal{B}(H)$, then

$$
K(A)=(R(S) \cap K(A)) \oplus(N(A) \cap K(A))
$$

Now, let $E$ another complex Hilbert space, called boundary space.

Definition 4.1. An operator $\Gamma: H \rightarrow E$ is said to be a boundary operator for a right generalized Drazin invertible operator $A$ corresponding to its right generalized Drazin inverse $S \in \mathcal{B}(H)$ if,

(i) $K(A) \subset N(\Gamma)$;

(ii) There exists an operator $\Pi: E \rightarrow H$ such that $\Gamma \Pi=I_{E}$ and $R(\Pi)=N(A) \cap K(A)$.

Theorem 4.1. Let $A \in \mathcal{B}(H)$ be a right generalized Drazin invertible operator with a right generalized Drazin inverse $S$. An operator $\Gamma: H \longrightarrow E$ is a boundary operator for $A$ corresponding to $S$ if and only if there exists a unique operator $\Pi: E \longrightarrow H$, as in the Definition 4.1, such that

$$
\Pi \Gamma x=x-S A x, \quad \text { for all } x \in K(A)
$$

Proof. Let $\Gamma: H \longrightarrow E$ be a boundary operator for $A$ corresponding to $S$, then there exists $\Pi: E \longrightarrow H$ satisfying the conditions of the Definition 4.1. Let $z=x-S A x$, then $x=z+S A x$, sinse $S A x \in R(S) \cap K(A)$ and $x \in K(A)$ we have $z \in N(A) \cap K(A)$, then $x-S A x \in R(\Pi)$, thus there exists $\varphi \in E$ such that $x-S A x=\Pi \varphi$. Since $N(\Gamma) \subset K(A)$ and $\Gamma \Pi=I_{E}$, we have $\Gamma(x-S A x)=\Gamma \Pi \varphi$ and $\varphi=\Gamma x$, which implies $(4.2)$.

The uniqueness of $\Pi$ is directly obtained.

Conversely, suppose that $\Gamma$ and $\Pi$ satisfies the identity (4.2). Then $A \Pi \Gamma x=0$, for all $x \in K(A)$, so $A \Pi=0$ on E. Moreover,

$$
\Pi \Gamma \Pi \Gamma x=\Pi \Gamma x-S A \Pi \Gamma x=\Pi \Gamma x .
$$

Hence $Г \Pi Г \Pi Г x=\Gamma \Pi \Gamma x$, so $\Gamma \Pi \varphi=\varphi$ for all $\varphi \in E$, by taking $\varphi=\Gamma \Pi \Gamma x$. Finally, we have $\Gamma S A x=$ $\Gamma x-\Gamma \Pi \Gamma x=0$. Thus $K(A) \subset N(\Gamma)$

Remark 4.1. If $\Gamma$ is a boundary operator for a right generalized Drazin invertible operator $A$ corresponding to its right generalized Drazin inverse $S$, then

$$
K(A)=(R(S) \cap K(A)) \oplus R(\Pi)
$$

Proposition 4.1 ( [15]). Let $A, B \in \mathcal{B}(H)$. Then $(I-\lambda A B)$ is invertible if and only if $(I-\lambda B A)$ is invertible for all $\lambda \neq 0$.

In this case, we have

$$
(I-\lambda B A)^{-1}=I+\lambda B(I-\lambda A B)^{-1} A
$$


and

$$
(I-\lambda A B)^{-1}=I+\lambda A(I-\lambda B A)^{-1} B
$$

Corollary 4.1. Let $A, B \in \mathcal{B}(H)$. If $\lambda^{-1} \in \rho(A B)$ then

$$
(I-\lambda A B)^{-1} A=A(I-\lambda B A)^{-1} .
$$

Proposition 4.2. Let $U_{L}=\left(\begin{array}{cc}T & L \\ 0 & D\end{array}\right)$ defined on $H \oplus K$. Assume that $S_{1}$ and $S_{2}$ are right generalized Drazin inverses of $T$ and $D$ respectively. $\Gamma_{1}$ and $\Gamma_{2}$ are boundary operators for $T$ and $D$ with the boundary spaces $E$ and $Z$; respectively. If $N(D) \subset N(L)$ then the operator $\Gamma=\left(\begin{array}{cc}\Gamma_{1} & 0 \\ 0 & \Gamma_{2}\end{array}\right)$ from $H \oplus K$ into $E \oplus Z$ is a boundary operator for $U_{L}$.

Proof. We have that $K(T) \subset N\left(\Gamma_{1}\right), K(D) \subset N\left(\Gamma_{2}\right)$ and there exist $\Pi_{1}: E \rightarrow H$ and $\Pi_{2}: Z \longrightarrow K$ such that $\Gamma_{1} \Pi_{1}=I_{E}, \quad R\left(\Pi_{1}\right)=N(T) \cap K(T)$ and $\Gamma_{2} \Pi_{2}=I_{Z}, \quad R\left(\Pi_{2}\right)=N(D) \cap K(D)$. Denote by $\Pi=\left(\begin{array}{ll}\Pi_{1} & 0 \\ 0 & \Pi_{2}\end{array}\right): E \oplus Z \longrightarrow H \oplus K$.

Since $T$ and $D$ are right generalized Drzain invertibles, then so is $U_{L}$, hence $K\left(U_{L}\right)=K(T) \oplus K(D)$, that is $K\left(U_{L}\right) \subset N(\Gamma)$ and $\Gamma \Pi=I_{E \oplus Z}$.

The condition $N(D) \subset N(L)$ implies that

$$
\begin{aligned}
N\left(U_{L}\right) \cap K\left(U_{L}\right) & =(N(T) \oplus N(D)) \cap(K(T) \oplus K(D)) \\
& =N(T) \cap K(T) \oplus N(D) \cap K(D)=R(\Pi) .
\end{aligned}
$$

Consider the operator $U_{L}$ defined as above and let $A \in \mathcal{B}(H)$ and $B \in \mathcal{B}(K)$ be given bounded operators on separable Hilbert spaces $H$ and $K$, and $M_{C}$ defined on $H \oplus K$ by (1.1). We define the following spectral boundary value matrix problem for unknown $w \in K(T) \times K(D)$ by

$$
\left\{\begin{array}{l}
\left(U_{L}-\lambda M_{C}\right) w=F \\
\Gamma w=\Phi
\end{array}\right.
$$

where $F \in K(T) \times K(D), \Phi \in E \times Z$ and $\lambda \in \mathbb{C}$ is a spectral parameter. We denote $\mathbf{R}_{\lambda}\left[S_{1} A\right]=\left(I_{H}-\lambda S_{1} A\right)^{-1}$ and $\mathbf{R}_{\lambda}\left[S_{2} A\right]=\left(I_{K}-\lambda S_{2} A\right)^{-1}, S_{1}$ and $S_{2}$ are right generalized Drazin inverses of $T$ and $D$, respectively.

Our purpose is to establish the existence and uniqueness of solutions for the boundary value problem $(\mathcal{P})$. In the theorem below, we give an explicit expression for the solution of the problem $(\mathcal{P})$. 
Theorem 4.2. If $\lambda^{-1} \in \rho\left(S_{1} A\right) \cap \rho\left(S_{2} B\right)$, the boundary value problem $(\mathcal{P})$ is uniquely solvable for any $F \in K(T) \times K(D)$ and $\Phi \in E \times Z$, the solution is given by

$$
w_{\lambda}^{F, \Phi}=G(S F+\Pi \Phi)
$$

where $S=\left(\begin{array}{cc}S_{1} & 0 \\ 0 & S_{2}\end{array}\right)$ and $G=\left(\begin{array}{cc}\mathbf{R}_{\lambda}\left[S_{1} A\right] & -S_{1} \mathbf{R}_{\lambda}\left[S_{1} A\right](L-\lambda C) \mathbf{R}_{\lambda}\left[S_{2} B\right] \\ 0 & \mathbf{R}_{\lambda}\left[S_{2} B\right]\end{array}\right)$.

Proof. We have $\left(U_{L}-\lambda M_{C}\right) w_{\lambda}^{F, \Phi}=\left(U_{L}-\lambda M_{C}\right) G S F+\left(U_{L}-\lambda M_{C}\right) G \Pi \Phi$. Firstly, we calculate $\left(U_{L}-\right.$ $\left.\lambda M_{C}\right) G S F$.

$$
\begin{aligned}
\left(U_{L}-\lambda M_{C}\right) G S F= & \left(U_{L}-\lambda M_{C}\right)\left(\begin{array}{cc}
\mathbf{R}_{\lambda}\left[S_{1} A\right] & -S_{1} \mathbf{R}_{\lambda}\left[S_{1} A\right](L-\lambda C) \mathbf{R}_{\lambda}\left[S_{2} B\right] \\
0 & \mathbf{R}_{\lambda}\left[S_{2} B\right]
\end{array}\right)\left(\begin{array}{c}
S_{1} f_{1} \\
S_{2} f_{2}
\end{array}\right) \\
= & \left(\begin{array}{cc}
(T-\lambda A) & (L-\lambda C) \\
0 & (D-\lambda B)
\end{array}\right)\left(\begin{array}{c}
\mathbf{R}_{\lambda}\left[S_{1} A\right] S_{1} f_{1}-S_{1} \mathbf{R}_{\lambda}\left[S_{1} A\right](L-\lambda C) \mathbf{R}_{\lambda}\left[S_{2} B\right] S_{2} f_{2} \\
\mathbf{R}_{\lambda}\left[S_{2} B\right] S_{2} f_{2}
\end{array}\right) \\
= & \left(\begin{array}{c}
(T-\lambda A) S_{1} \mathbf{R}_{\lambda}\left[A S_{1}\right] f_{1} \\
(D-\lambda B) S_{2} \mathbf{R}_{\lambda}\left[B S_{2}\right] f_{2}
\end{array}\right)=F,
\end{aligned}
$$

and

$$
\begin{aligned}
\left(U_{L}-\lambda M_{C}\right) G \Pi \Phi= & \left(\begin{array}{c}
(T-\lambda A)\left[\mathbf{R}_{\lambda}\left[S_{1} A\right] \Pi_{1} \varphi_{1}-S_{1} \mathbf{R}_{\lambda}\left[S_{1} A\right](L-\lambda C) \mathbf{R}_{\lambda}\left[S_{2} B\right] \Pi_{2} \varphi_{2}\right] \\
+(L-\lambda C) \mathbf{R}_{\lambda}\left[S_{2} B\right] \Pi_{2} \varphi_{2} \\
(D-\lambda B) \mathbf{R}_{\lambda}\left[S_{2} B\right] \Pi_{2} \varphi_{2}
\end{array}\right) \\
= & \left(\begin{array}{c}
(T-\lambda A) \mathbf{R}_{\lambda}\left[S_{1} A\right] \Pi_{1} \varphi_{1} \\
(D-\lambda B) \mathbf{R}_{\lambda}\left[S_{2} B\right] \Pi_{2} \varphi_{2}
\end{array}\right) \\
= & \left(\begin{array}{l}
(T-\lambda A)\left[I_{H}+\lambda S_{1} \mathbf{R}_{\lambda}\left[A S_{1}\right] A\right] \Pi_{1} \varphi_{1} \\
(D-\lambda B)\left[I_{K}+\lambda S_{2} \mathbf{R}_{\lambda}\left[B S_{2}\right] B\right] \Pi_{2} \varphi_{2}
\end{array}\right) \\
= & \left(\begin{array}{c}
(T-\lambda A) \Pi_{1} \varphi_{1}+\lambda A \Pi_{1} \varphi_{1} \\
(D-\lambda B) \Pi_{2} \varphi_{2}+\lambda B \Pi_{2} \varphi_{2}
\end{array}\right) .
\end{aligned}
$$


Then $\left(U_{L}-\lambda M_{C}\right) G \Pi \Phi=0$ since $R\left(\Pi_{1}\right)=N(T) \cap K(T)$ and $R\left(\Pi_{2}\right)=N(D) \cap K(D)$. Using the fact that $K(T) \subset N\left(\Gamma_{1}\right)$ and $K(D) \subset N\left(\Gamma_{2}\right)$, we obtain,

$$
\begin{aligned}
\Gamma w_{\lambda}^{F, \Phi}= & \Gamma G(S F+\Pi \Phi) \\
= & \left(\begin{array}{cc}
\Gamma_{1} & 0 \\
0 & \Gamma_{2}
\end{array}\right)\left(\begin{array}{c}
\mathbf{R}_{\lambda}\left[S_{1} A\right] S_{1} f_{1}-S_{1} \mathbf{R}_{\lambda}\left[S_{1} A\right](L-\lambda C) \mathbf{R}_{\lambda}\left[S_{2} B\right] S_{2} f_{2} \\
\mathbf{R}_{\lambda}\left[S_{2} B\right] S_{2} f_{2}
\end{array}\right) \\
& +\left(\begin{array}{cc}
\Gamma_{1} & 0 \\
0 & \Gamma_{2}
\end{array}\right)\left(\begin{array}{c}
\mathbf{R}_{\lambda}\left[S_{1} A\right] \Pi_{1} \varphi_{1}-S_{1} \mathbf{R}_{\lambda}\left[S_{1} A\right](L-\lambda C) \mathbf{R}_{\lambda}\left[S_{2} B\right] \Pi_{2} \varphi_{2} \\
\mathbf{R}_{\lambda}\left[S_{2} B\right] \Pi_{2} \varphi_{2}
\end{array}\right) \\
& =\left(\begin{array}{c}
\Gamma_{1} \mathbf{R}_{\lambda}\left[S_{1} A\right] \Pi_{1} \varphi_{1}-\Gamma_{1} S_{1} \mathbf{R}_{\lambda}\left[S_{1} A\right](L-\lambda C) \mathbf{R}_{\lambda}\left[S_{2} B\right] \Pi_{2} \varphi_{2} \\
\Gamma_{2} \mathbf{R}_{\lambda}\left[S_{2} B\right] \Pi_{2} \varphi_{2}
\end{array}\right) \\
= & \left(\begin{array}{c}
\Gamma_{1}\left[I_{H}+\lambda S_{1} \mathbf{R}_{\lambda}\left[A S_{1}\right] A\right] \Pi_{1} \varphi_{1} \\
\Gamma_{2}\left[I_{K}+\lambda S_{2} \mathbf{R}_{\lambda}\left[B S_{2}\right] B\right] \Pi_{2} \varphi_{2}
\end{array}\right) \\
= & \left(\begin{array}{c}
\Gamma_{1} \Pi_{1} \varphi_{1} \\
\Gamma_{2} \Pi_{2} \varphi_{2}
\end{array}\right)=\Phi .
\end{aligned}
$$

The uniqueness of the solution of $(\mathcal{P})$ follows from standard arguments. That is, if $w_{1}, w_{2} \in K(T) \times K(D)$ are two solutions of $(\mathcal{P})$, assume that $w_{0}=w_{1}-w_{2}=\left(\begin{array}{c}u_{0} \\ v_{0}\end{array}\right)=\left(\begin{array}{c}S_{1} f_{0}+\Pi_{1} \varphi_{0} \\ S_{2} g_{0}+\Pi_{2} \psi_{0}\end{array}\right)$ with some $f_{0} \in$ $K(T), g_{0} \in K(D), \varphi_{0} \in E$ and $\psi_{0} \in Z$. Thus,

$$
\left\{\begin{array}{l}
\left(U_{L}-\lambda M_{C}\right) w_{0}=0 \\
\Gamma w_{0}=0
\end{array}\right.
$$

Since $K\left(U_{L}\right) \subset N(\Gamma)$ and $\Gamma \Pi=I_{E \oplus Z}$, the second identity gives $\left(\begin{array}{l}\varphi_{0} \\ \psi_{0}\end{array}\right)=\left(\begin{array}{l}0 \\ 0\end{array}\right)$. Then $u_{0}=S_{1} f_{0}$ and $v_{0}=S_{2} g_{0}$. So,

$$
\begin{aligned}
0=\left(U_{L}-\lambda M_{C}\right) w_{0} & =\left(\begin{array}{cc}
(T-\lambda A) & (L-\lambda C) \\
0 & (D-\lambda B)
\end{array}\right)\left(\begin{array}{c}
S_{1} f_{0} \\
S_{2} g_{0}
\end{array}\right) \\
& =\left(\begin{array}{c}
(T-\lambda A) S_{1} f_{0}+(L-\lambda C) S_{2} g_{0} \\
(D-\lambda B) S_{2} g_{0}
\end{array}\right) .
\end{aligned}
$$

Then, $f_{0}=g_{0}=0$, since $\lambda^{-1} \in \rho\left(S_{1} A\right) \cap \rho\left(S_{1} B\right), f_{0} \in K(T)$ and $g_{0} \in K(D)$. Hence $w_{1}=w_{2}$. This complete the proof. 


\section{REFERENCES}

[1] P. Aiena, Fredholm and local spectral theory, with applications to multipliers, Kluwer Academic Publishers, 2004.

[2] M. Barraa and M. Boumazgour, On the perturbations of spectra of upper triangular operator matrices, J. Math. Anal. Appl. 347 (1) (2008), 315-322.

[3] M. Benharrat and B. Messirdi, On the generalized Kato spectrum, Serdica Math. J. 37 (4) (2011), 283-294.

[4] M. Boumezgour, Drazin invertibility of upper triangular operator matrices, Linear and Multilinear Algebra 61 (5) (2013) , 627-634.

[5] X. H. Cao, M. Z. Guo and B. Meng, Drazin spectrum and Weyl's theorem for operator matrices, J. Math. Res. Exposition, 26 (3) (2006), 413-422.

[6] S. V. Djordjevic and B. P. Duggal, Drazin invertibility of the diagonal of an operator, Linear and Multilinear Algebra, 60 (1) (2012), 65-71.

[7] H. K. Du and J. Pan, Perturbation of spectrum of $2 \times 2$ operator matrices, Proc. Amer. Math. Soc. 121 (3) (1994), 761-766.

[8] S. Grabiner, Ascent, descent, and compact perturbations, Proc. Amer. Math. Soc. 71 (1) (1978), 79-80.

[9] J. K. Han, H. Y. Lee and W. Y. Lee, Invertible completions of $2 \times 2$ upper triangular operator matrices, Proc. Amer. Math. Soc. 128 (1) (1999), 119-123.

[10] G.L. Han and V. Chen, On the right (left) invertible completions for operator matrices, Integr. Equ. Oper. Theory 67 (1) (2010), 79-93.

[11] G. L. Han and A. Chen, Perturbations of the right and left spectra for operator matrices, J. Oper. Theory 67 (1) (2012), $207-214$.

[12] R. Harte, On Kato non-singularity, Studia Math. 117 (2) (1996), 107-114.

[13] I. S. Hwang and W. Y. Lee, The boundedness below of $2 \times 2$ upper triangular operator matrices, Integr. Equ. Oper. Theory 39 (3) (2001), 267-276.

[14] M. A. Kaashoek and D.C. Lay, Ascent, descent, and commuting perturbations, Trans. Amer. Math. Soc. 169 (1972), 35-47.

[15] N. Khaldi, M. Benharrat and B. Messirdi, On the Spectral Boundary Value Problems and Boundary Approximate Controllability of Linear Systems. Rend. Circ. Mat. Palermo 63 (1) (2014), 141-153.

[16] N. Khaldi, M. Benharrat and B. Messirdi, A spectral analysis for solving boundary value matrix problems: existence, uniqueness and application to symplectic elasticity, J. Adv. Res. Appl. Math. 6 (4) (2014), 68-80.

[17] Kato, T., Perturbation theory for linear operators, Springer-Verlag, New York, 1966.

[18] J. J. Koliha, A generalized Drazin inverse, Glasgow Math. J. 38 (3) (1996), 367-381,

[19] V. Kordula and V. Müller, The distance from the Apostol spectrum, Proc. Amer. Math. Soc. 124 (10) (1996), $3055-3061$.

[20] J-P. Labrousse, Les opérateurs quasi-Fredholm une généralisation des opérateurs semi-Fredholm, Rend. Circ. Mat. Palermo 29 (1) (1980), 161-258.

[21] W. Y. Lee, Weyl spectra of operator matrices, Proc. Amer. Math. Soc. 129 (1) (2001), 131-138.

[22] Y. Li, X. H. Sun, and H. K. Du, The intersection of left (right) spectra of $2 \times 2$ upper triangular operator matrices, Linear Algebra Appl. 418 (1) (2006), 112-121.

[23] M. Mbekhta, Opérateurs pseudo-Fredholm I : Résolvant généralisé, J. Operator Theory 24 (2) ( 1990), $255-276$.

[24] K. Miloud Hocine, M. Benharrat and B. Messirdi, Left and right generalized Drazin invertible operators, Linear and Multilinear Algebra 63 (8) (2015), 1635-1648.

[25] V. Müller, On the regular spectrum, J. Operator Theory 31 (2) (1994), 363-380.

[26] V. Müller, Spectral theory of linear operators and spectral systems in Banach algebra, Birkhauser, 2007. 
[27] V. Rakočević, Generalized spectrum and commuting compact perturbations, Proc. Edinburgh. Math. Soc.36 (2) (1993), 197-209.

[28] S. F. Zhang, H. J. Zhong, and Q. F. Jiang, Drazin spectrum of operator matrices on the Banach space, Linear Algebra Appl. 429 (8-9) (2008), 2067-2075.

[29] Y. N. Zhang, H. J. Zhong and L. Q. Lin, Browder spectra and essential spectra of operator matrices, Acta Math. Sinica 24 (6) (2008), 947-954.

[30] S. F. Zhang, H. J. Zhong and L. Q. Lin, Generalized Drazin spectrum of operator matrices, Appl. Math. J. Chinese Univ. 29 (2) (2014), 162-170. 